\title{
Infección tuberculosa latente en personal de salud de las Américas
}

\author{
DOI: https://doi.org/10.5377/alerta.v3i1.8844
}

\author{
Josué Bernabé Villalobos Montoya ${ }^{1^{*}}$, Juan Gabriel Cisneros García ${ }^{2}$, Mauricio Alexander Juárez Alvarado ${ }^{3}$, \\ José Carlos Henríquez Sosa ${ }^{4}$
}

1. Doctor en medicina, Máster en salud pública, coordinador, Unidad Comunitaria de Salud Familiar Especializada de Dulce Nombre de María, Ministerio de Salud, Municipio de Dulce Nombre de María, Chalatenango, El Salvador.

2. Doctor en medicina, Máster en salud pública, Máster en infecciones de transmisión sexual y VIH/sida, colaborador técnico médico, Región central de salud, Ministerio de Saud, Santa Tecla, La Libertad, Minsal, El Salvador.

3. Doctor en medicina, especialista en medicina familiar, colaborador técnico médico, Unidad de desarrollo de servicios de salud, Dirección del primer nivel de atención, Ministerio de Salud, San Salvador, El Salvador.

4. Doctor en medicina, director, Unidad Comunitaria de Salud Familiar Especializada del Puerto de la Libertad, Ministerio de Salud, La libertad, El Salvador.

${ }^{*}$ Correspondencia

$\square$ dr.josuevillalobos@gmail.com

(1D) 0000-0003-4061-1677

\section{Resumen}

Introducción. Según la Organización Mundial para la Salud, un tercio de la población mundial tiene tuberculosis latente, considerando al personal de salud como uno de los grupos de riesgo con mayor tasa de prevalencia. Objetivo. En el presente estudio se realizó una revisión bibliográfica de la evidencia existente acerca de la situación epidemiológica de la infección tuberculosa latente en trabajadores de salud en las Américas. Metodología. Este estudio comprende una revisión sistemática de estudios transversales, metaanálisis y de cohorte, publicados en los últimos 10 años. Se seleccionaron los estudios que cumplieron con criterios de inclusión y de calidad. Resultados. Se seleccionaron 15 de 288 estudios encontrados, pertenecientes a 8 países de América. La prevalencia oscilaba entre 15\% y 62.1\%. La prevalencia de infección tuberculosa latente en nueve estudios es mayor a la de la población global, en cuatro es similar y en uno es inferior. Conclusión. La prevalencia de tuberculosis sigue siendo mayor en los trabajadores de salud que en la población general. Esto afecta mayoritariamente a las mujeres entre los 35 y 43 años que han laborado por más de 10 años en centros penitenciarios o centros de atención primaria.

\section{Palabras clave}

Tuberculosis latente, personal de salud, Américas.

\begin{abstract}
Introduction. According to the World Health Organization, a third of the world's population has latent tuberculosis, considering health personnel as a risk group, with a higher prevalence rate. Objective. A bibliographic review of the existing evidence on the epidemiological situation of latent tuberculosis infection in health workers in the Americas was carried out. Methods. This study includes a systematic review of cross-sectional, meta-analysis and cohort studies, published in the last 10 years. The studies that met the inclusion and quality criteria were selected. Results. Fifteen of the 288 studies found in eight countries in the Americas were selected, with prevalence ranging from $15 \%$ to $62.1 \%$. The prevalence of latent tuberculosis infection in nine studies is higher than the prevalence in the global population, in four it is similar and one is lower. Conclusion. The prevalence of tuberculosis remains higher in health workers than in the general population. This mostly affects women between the ages of 35 and 43 who have worked for more than 10 years in prisons or primary care centers.
\end{abstract}

\section{Keywords}

Latent tuberculosis, health personnel, Americas.

\section{ACCESO ABIERTO}

\section{Latent tuberculosis \\ infection in health}

personnel in America

Citación recomendada:

Villalobos Montoya JB, Cisneros García JG, Juárez Alvarado MA,

Henríquez Sosa JC.

Alerta 2020; 3(1):23-32

DOI: https://doi.org/10.5377/ alerta.v3i1.8844

\section{Recibido:}

29 de noviembre 2019

\section{Aceptado:}

18 de enero de 2020

Publicado:

27 de enero de 2020

\section{Contribución de autoría:}

JBVM': Organización del

proceso de revisión. JBVM ${ }^{\top}$

$\mathrm{JGCG}^{2}, \mathrm{MAJA}^{3}, \mathrm{JCHS}^{4}$

Búsqueda de datos, selección

de artículos, análisis y

discusión de datos.

\section{Conflicto de intereses:}

Los autores no tienen conflicto de intereses. 


\section{Introducción}

La tuberculosis (TB) es una de las primeras causas de mortalidad a nivel mundial entre las enfermedades infecciosas' ${ }^{1}$. Cada año aparecen 280000 casos nuevos en la región de las Américas, de los cuales se estima que fallecen $23000^{2}$.

Según la Organización Mundial de la Salud, un tercio de la población mundial son portadores asintomáticos del bacilo causante de la tuberculosis ${ }^{3}$. Esta condición es conocida como infección tuberculosa latente (ITBL) y se define como un estado de respuesta inmunitaria persistente a antígenos de Mycobacterium tuberculosis, adquiridos con anterioridad que no se acompaña de manifestaciones clínicas de tuberculosis activa 4 .

En el 2010 la Organización Internacional del Trabajo integró a la TB dentro del listado de enfermedades ocupacionales 5 . El contacto frecuente con personas con TB es un factor de riesgo para adquirir la enfermedad ${ }^{6}$, esto representa un alto riesgo para los trabajadores de salud ${ }^{7}$. Diferentes estudios ${ }^{8-10}$ han demostrado que el riesgo de desarrollar TB en los trabajadores de salud (37\%-63\%) es mayor que el de la población general (30\%).

Entre los factores de riesgo para el desarrollo de la TB se consideran: edad mayor de 50 años, estado de exfumador auto afirmado, personal de salud, uso irregular de la mascarilla N95 ${ }^{11}$, un mayor tiempo de laborar en el establecimiento, entre otros ${ }^{12}$. Conocer la situación de la ITBL es prioritario, ya que estas personas en riesgo de contraer la TB deben ser priorizadas para implementar estrategias de tratamiento. Por tal razón, y tomando en cuenta que los trabajadores de salud son un grupo que se encuentra en riesgo de adquirir la enfermedad, se realizó una revisión bibliográfica de la evidencia disponible de la situación epidemiológica de la tuberculosis latente en los trabajadores de salud en América y sus factores de riesgo.

\section{Metodología}

\section{Búsqueda}

Se realizó una revisión sistemática de publicaciones en las siguientes bases de datos electrónicas: PUBMED, LILACS, Google Académico y en páginas de literatura gris. Se utilizaron los siguientes descriptores (palabras claves): latent tuberculosis, health personnel, health care workers, epidemiology, tuberculosis latente, epidemiología y personal de salud. Posteriormente la información fue organizada en una tabla.
Se incluyeron artículos originales de estudios transversales y cohortes, revisiones sistemáticas y metaanálisis, que se publicaron en el período de enero enero 2009 a octubre 2019. Se incluyeron estudios en inglés, portugués y español que tuvieran como población los trabajadores de salud, independientemente del género, sexo, edad o disciplina. Finalmente, se escogieron aquellos estudios que se realizaron en la región de las Américas. Algunos estudios que se encontraron de manera incidental y que se consideraron relevantes también fueron seleccionados para la revisión.

No se consideraron para la revisión los estudios cualitativos, recomendaciones de expertos, guías de práctica clínica, noticias de periódicos, comunicados de asociación de pacientes o personas afectadas.

\section{Selección de estudio}

Posterior a la realización de la estrategia de búsqueda, se cumplieron los siguientes pasos: lectura de títulos de los estudios relacionados con la pregunta de investigación, a aquellos que eran relevantes y contenían los elementos PICO se les realizó una lectura crítica utilizando la escala de New Castle Ottawa. Se organizaron en una matriz de síntesis todos los estudios que cumplían con los criterios de calidad y que presentaron resultados relevantes para el objetivo de estudio.

\section{Resultados}

Según las estrategias de búsqueda implementadas, se encontraron un total de 288 artículos relacionados a tuberculosis latente, 285 en bases de datos y 3 de otras búsquedas consideradas como literatura gris a través de Google Académico. De las búsquedas en bases de datos, en PubMed se encontraron $172(59.7 \%)$, LILACS 71 (24.6\%) y 42 en BVS (14.6\%). Relacionados con la pregunta de investigación se encontraron 28 , de los cuales 2 estaban repetidos en dos de las bases utilizadas; se excluyeron 11 por no cumplir con los criterios de selección, quedando un total 15 artículos para el análisis (Figura 1).

La Tabla 1 resume el total de estudios que se incluyeron para esta revisión. Fueron 9098 las personas representadas en las investigaciones de los 15 artículos revisados. De las publicaciones seleccionadas, 13 fueron estudios transversales, uno fue estudio de cohorte y se encontró un metaanálisis, todos ellos realizados en 8 países de la región de las Américas. El país con más cantidad de artículos considerados para esta revisión fue Brasil, con 7 (46.6\%), de Cuba 


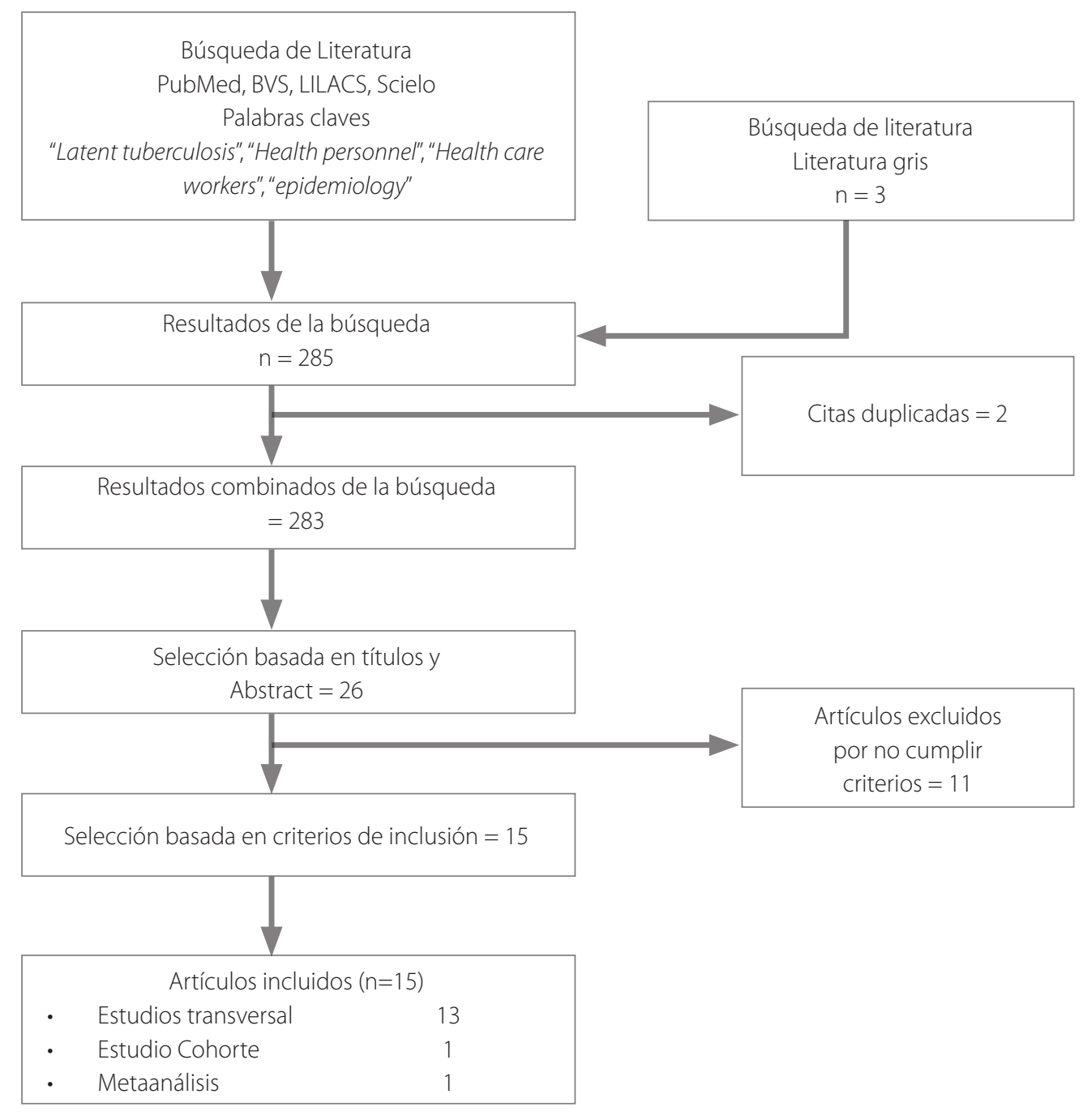

Figura 1. Proceso de selección de estudios para análisis

fueron 2 (13.3\%) y de los demás, Argentina, Canadá, Chile, Colombia, Perú y USA, una publicación en esta revisión.

En diez de los estudios se utilizó prueba de derivado proteico para reportar la ITBL; otros tres utilizaron IGRA, en uno de los estudios utilizaron ambas ${ }^{13}$ y únicamente en el estudio de Bussato ${ }^{14}$ se describe la prevalencia de ITBL de acuerdo con el auto reporte de cada participante en la encuesta.

El metaanálisis de Nasreen et al. ${ }^{15}$, realizado en 2016, consideró 18 estudios para su revisión, incluyendo países como Bangladesh, China, India, Sudáfrica, Uganda, Zimbabue y Brasil, siendo este último el único país de América que fue incluido y a quien perteneció la tercera parte de los estudios revisados en este metaanálisis. La prevalencia descrita para Brasil en este documento es la que se tomó en cuenta para el presente artículo de revisión bibliográfica.

La prevalencia de la ITBL en América ha sido descrita desde un $15 \%$ hasta un $62.1 \%$ (Figura 1). Bussato et al., $2017^{14}$ describió la tasa más baja de ITBL en trabajadores de sa- lud (15\%), con la característica que en este estudio la prevalencia se calculó a partir del auto reporte del resultado de la prueba de derivado proteico de cada participante, si es que se le había realizado en algún momento de la vida laboral, independientemente del tiempo transcurrido desde que se realizó la prueba. La Tabla 2 resume la metodología y los hallazgos más relevantes de cada estudio incluidos en esta revisión.

Los factores de riesgo para la ITBL más frecuentemente observados en trabajadores de salud fueron: el sexo femenino ${ }^{15-19}$, la edad del trabajador entre 35 y 43 años $^{15-19}$ y la presencia de cicatriz de $B C G^{11,18}$.

La ITBL descrita en los estudio incluyó a diferentes niveles de atención. Por ejemplo, Bussato et al. ${ }^{14}$ investigó en personal de salud de centros penitenciarios; Prado ${ }^{11}$, Lacer$\mathrm{da}^{19}$, Soto Cabezas $^{12}$ y Borges $^{17}$, en centros de atención primaria; Rogerio ${ }^{20}$ en personal comunitario; el resto en personal hospitalario. Gutiérrez et al. ${ }^{16}$ reportó una mayor frecuencia en enfermeras (64.7\%) y el menor porcentaje en personal técnico (30\%). 
Entre los estudios que se revisaron se observa cómo la prevalencia aumenta en personal que ha laborado por más de 10 años, con una prevalencia entre el $60 \%$ y $63 \% 15,20$ y en aquellos con más de 35 años de laborar con una prevalencia entre $58 \%$ y $60 \%{ }^{20}$. En cuanto a fumadores, solo dos estudios describen una asociación estadísticamente significativa ${ }^{17,19}$.

\section{Discusión}

En general, la prevalencia de ITBL en profesionales de salud de estos países oscilaba entre $15 \%$ y $62.1 \%$. El país que reportó una prevalencia mayor en los trabajadores de salud fue Colombia' ${ }^{19}$, que superaba el $60 \%$. El estudio que reportó una menor prevalencia fue uno realizado en los trabajadores de salud que laboraban en centros penales de Brasil ${ }^{14}$; sin embargo, es necesario aclarar que la prevalencia obtenida en este estudio fue auto reportada por el personal de salud, a quienes se les consultó si alguna vez se les había realizado la prueba de derivado proteico en su tiempo de trabajo y cuál había sido el resultado. El promedio de tiempo laboral era de 15 años, por lo cual existe un riesgo de sesgo de memoria en el resultado publicado. Con esto no se observa una relación entre la prevalencia de ITBL y TB activa, ya que a pesar de que Colombia y Perú tienen las prevalencias de ITBL más elevadas, es Brasil el país con mayor carga de TB en las Américas.
En un metaanálisis que estimó la prevalencia global de ITLB a partir de 88 estudios de 36 países, publicados entre 2005 a 2018 (Cohen et al) ${ }^{21}$, la prevalencia de ITBL con prueba de interferón gamma fue de $24.8 \%$ (IC95 \% 19.7-29.9\%) y con PPD fue de $21.2 \%$ (IC95\% 17.9-24.2\%). Al comparar estos resultados con lo encontrado en esta revisión, se observó que del total de publicaciones nueve de ellas reportaron prevalencias mayores a la estimada en esa revisión sistemática, cuatro publicaciones tenían prevalencias similares y solamente una de ellas tenía una prevalencia menor. Sin embargo, esta última fue el estudio que obtuvo la prevalencias a partir del auto reporte.

Al realizar la comparación entre las dos revisiones sistemáticas, se observó que la carga de ITBL sigue siendo mayor en personal de salud en comparación con la población general, ya que la revisión sistemática y metaanálisis de la prevalencia de ITBL en trabajadores de salud de países con más carga por TB, obtuvo una prevalencia global de $47 \%$ (IC95\% 34-60) y en Brasil de 37\% (IC95\% 17-56), siendo mucho mayor de lo reportado por Cohen ${ }^{21}$.

Los estudios revisados muestran que enfermería es la disciplina con mayor porcentaje de ITBL y el personal médico con la menor. Esto puede obedecer a que las enfermeras son las que tienen mayor contacto con los pacientes infectados con TB para realizar educación en salud, administrar tratamiento, cuidados generales, entre otros.

Tabla 1. Listado de publicaciones incluida en la revisión bibliográfica

\begin{tabular}{lllll}
\hline Autor & País & Año & $\begin{array}{l}\text { No de } \\
\text { participantes }\end{array}$ & Diseño del estudio \\
\hline Bussato et al. & Brasil & 2017 & 114 & Estudio transversal \\
Prado et al. & Brasil & 2017 & 708 & Estudio transversal \\
Rogerio, Wesley Pereira & Brasil & 2015 & 321 & Estudio transversal \\
Gutiérrez y Molina & Cuba & 2012 & 183 & Estudio transversal \\
Gutiérrez y Molina & Cuba & 2015 & 818 & Estudio transversal \\
Couto, Ingrid & Brasil & 2014 & 663 & Estudio transversal \\
Hernández, Mariluz & Chile & 2014 & 76 & Estudio transversal \\
Silvana Borges & Brasil & 2011 & 137 & Estudio transversal \\
Soto Cabezas, M. & Perú & 2017 & 150 & Estudio transversal \\
Bavaresco et al. & Brasil & 2017 & 225 & Estudio transversal \\
Nasreen et al. & Canadá & 2016 & 2670 & Metaanálisis \\
Lacerda et al. & Brasil & 2012 & 218 & Estudio transversal \\
González et al. & Argentina & 2010 & 670 & Estudio transversal \\
Ochoa et al. & Colombia & 2016 & 1218 & Estudio transversal \\
Arguello Pérez & USA & 2017 & 927 & Estudio Cohorte \\
\hline
\end{tabular}




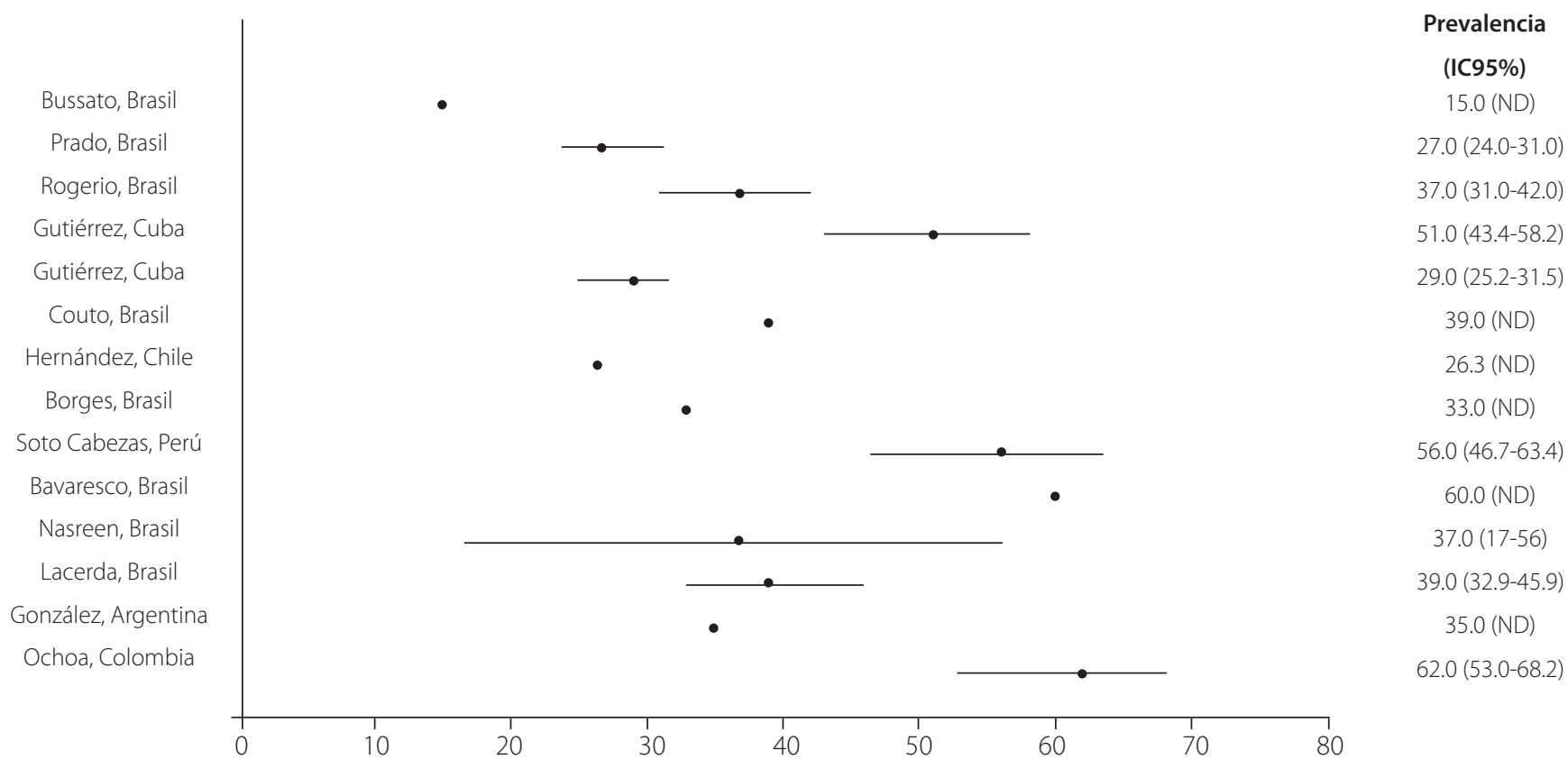

Figura 1. Prevalencia de infección tuberculosa latente reportadas en América en los últimos 10 años

Tabla 2. Resumen y metodología de los estudios de ITBL en América, 2009 a octubre 2019

\begin{tabular}{|c|c|c|c|c|c|c|}
\hline N. ${ }^{\circ}$ & Autor & Año & Título & Objetivo & Metodología & Limitantes/Posibles sesgos \\
\hline 1 & Busatto et al. & 2017 & $\begin{array}{l}\text { Tuberculo- } \\
\text { sis among } \\
\text { prison staff in Río } \\
\text { Grande do Sul. }\end{array}$ & $\begin{array}{l}\text { Evaluar el riesgo } \\
\text { de infección y de } \\
\text { la enfermedad } \\
\text { por Mycobac- } \\
\text { terium tubercu- } \\
\text { losis entre los } \\
\text { profesionales de la } \\
\text { salud y seguridad } \\
\text { en los centros pe- } \\
\text { nitenciarios en dos } \\
\text { regiones del estado } \\
\text { de Rio Grande do } \\
\text { Sul (RS). }\end{array}$ & $\begin{array}{l}\text { Estudio Observacional, } \\
\text { descriptivo en } 114 \\
\text { profesionales, entre } \\
\text { personal de asistencia } \\
\text { sanitaria y seguridad de } \\
4 \text { cárceles en } 2 \text { regiones } \\
\text { distintas del estado de } \\
\text { Rio Grande do Sul. } \\
\text { La estimación de la pre- } \\
\text { valencia fue a través del } \\
\text { auto reporte del resulta- } \\
\text { do de la última PPD que } \\
\text { se había realizado a los } \\
\text { participantes } \\
\text { Limitante: Posible sesgo } \\
\text { de memoria, debido a } \\
\text { que la estimación de } \\
\text { prevalencia fue a través } \\
\text { del auto reporte. }\end{array}$ & $\begin{array}{l}\text { La prevalencia de la TB latente fue del } 27.9 \% \text {. El } \\
\text { tiempo de trabajo entre las diferentes categorías } \\
\text { profesionales y la región en la que trabajan fueron } \\
\text { considerados factores de riesgo para la TB latente. }\end{array}$ \\
\hline 2 & Prado et al. & 2017 & $\begin{array}{l}\text { Prevalencia } \\
\text { y factores de } \\
\text { riesgo de ITBL en } \\
\text { trabajadores de } \\
\text { salud de aten- } \\
\text { ción primaria en } \\
\text { Brasil. }\end{array}$ & $\begin{array}{l}\text { Determinar la pre- } \\
\text { valencia y factores } \\
\text { de riesgo para } \\
\text { ITBL entre PS de } \\
\text { atención primaria } \\
\text { en } 5 \text { ciudades } \\
\text { brasileñas. }\end{array}$ & $\begin{array}{l}\text { Se realizó un estudio } \\
\text { transversal, de } 2011 \text { a } \\
2013 \text { en trabajadores } \\
\text { de salud primarios, a } \\
\text { través de un cuestiona- } \\
\text { rio estructurado y una } \\
\text { evaluación de la LTBI } \\
\text { mediante la prueba de } \\
\text { IGRA. }\end{array}$ & $\begin{array}{l}\text { Factores de riesgo identificados: edad >50 años, } \\
\text { ausencia de cicatriz de BCG, auto reportados exfu- } \\
\text { madores, ser enfermera, ser técnico de enfermería, } \\
\text { ser PS, uso irregular de respirador N95. }\end{array}$ \\
\hline
\end{tabular}


Tabla 2. Continuación.

\begin{tabular}{|c|c|c|c|c|c|c|}
\hline 3 & Rogerio et al. & 2015 & $\begin{array}{l}\text { Prevalencia y fac- } \\
\text { tores asociados a } \\
\text { la infección por } \\
\text { M. tuberculosis, } \\
\text { usando la prue- } \\
\text { ba de tuberculi- } \\
\text { na entre agentes } \\
\text { comunitarios de } \\
\text { salud en Brasil. }\end{array}$ & $\begin{array}{l}\text { Determinar la } \\
\text { prevalencia y los } \\
\text { factores de riesgo } \\
\text { asociados a la } \\
\text { infección por M. } \\
\text { tuberculosis entre } \\
\text { agentes comunita- } \\
\text { rios de salud. }\end{array}$ & $\begin{array}{l}\text { Estudio transversal en } \\
322 \text { trabajadores de } \\
\text { salud donde se indagó } \\
\text { factores de riesgo e } \\
\text { ITBL a través de un } \\
\text { cuestionario y PPD en } \\
\text { el año } 2011 \text { y } 2012 \text {. Se } \\
\text { analizó la prevalencia } \\
\text { en un corte de } 5 \text { mm y } \\
10 \text { mm. }\end{array}$ & $\begin{array}{l}\text { De acuerdo con los puntos de corte, el } 57.88 \% \\
\text { (IC95\%; 52,0-63,0) fue positivo a } 5 \text { mm y el 37.3\% } \\
\text { (IC95\%; 31,0-42,0) fue positivo a } 10 \mathrm{~mm} \text {. No se } \\
\text { obtuvo ninguna asociación entre la frecuencia de } \\
\text { acceso al conocimiento de TB y positividad a PT. }\end{array}$ \\
\hline 4 & Borroto et al. & 2012 & $\begin{array}{l}\text { Riesgo de ocu- } \\
\text { rrencia de TB en } \\
\text { los trabajadores } \\
\text { del Hospital } \\
\text { Universitario } \\
\text { Neumológico } \\
\text { Benéfico Jurídico } \\
\text { de La Habana. }\end{array}$ & $\begin{array}{l}\text { Evaluar el riesgo in- } \\
\text { dividual y colectivo } \\
\text { de infección tuber- } \\
\text { culosa por áreas o } \\
\text { departamentos en } \\
\text { el Hospital Neumo- } \\
\text { lógico Universitario } \\
\text { Benéfico Jurídico. }\end{array}$ & $\begin{array}{l}\text { Durante 2008- } 2009 \\
\text { se midió el riesgo de } \\
\text { tuberculosis para los } \\
\text { trabajadores; se aplicó } \\
\text { una encuesta con datos } \\
\text { personales, de ubica- } \\
\text { ción laboral y exposi- } \\
\text { ción al Mycobacterium } \\
\text { tuberculosis, así como } \\
\text { una prueba tuberculí- } \\
\text { nica a } 112 \text { de ellos y se } \\
\text { consideraron positivas } \\
\text { aquellas } \geq 10 \text { mm. }\end{array}$ & $\begin{array}{l}\text { La prevalencia de reactores a la PPD en la presente } \\
\text { encuesta resultó de } 30.1 \% \text { La mayor prevalencia de } \\
\text { reactores se encontró en los trabajadores de más } \\
\text { de } 20 \text { años laborando en la institución ( } 60.5 \%) \text { y } \\
\text { la menor en el grupo de menos de } 1 \text { año }(40.9 \%) \\
\text { pero sin diferencia significativa entre las categorías } \\
(p=0,62) \text {. }\end{array}$ \\
\hline 5 & Borroto et al. & 2015 & $\begin{array}{l}\text { Riesgo de TB en } \\
\text { trabajadores de } \\
3 \text { hospitales clí- } \\
\text { nicos quirúrgicos } \\
\text { de La Habana. }\end{array}$ & $\begin{array}{l}\text { Estimar la prevalen- } \\
\text { cia de ITBL y evaluar } \\
\text { el riesgo de infec- } \\
\text { ción tuberculosa en } \\
\text { trabajadores de tres } \\
\text { hospitales clínicos } \\
\text { Quirúrgicos de La } \\
\text { Habana, Cuba. }\end{array}$ & $\begin{array}{l}\text { Estudio observacional, } \\
\text { descriptivo en } 804 \\
\text { trabajadores de } 3 \text { hospi- } \\
\text { tales de la Habana. } \\
\text { Se les aplicó prueba de } \\
\text { tuberculina y cuestio- } \\
\text { nario. }\end{array}$ & $\begin{array}{l}\text { La prevalencia de ITBL fue de 28,8\%. El riesgo de In- } \\
\text { fección por M. Tuberculosis para los trabajadores de } \\
\text { los tres hospitales clínico-quirúrgicos de La Habana } \\
\text { fue evaluado entre intermedio y alto, con un tercio } \\
\text { de sus áreas que constituyen un potencial de riesgo } \\
\text { de tuberculosis para el personal que labora en ellas. }\end{array}$ \\
\hline 6 & Ramos et al. & 2014 & $\begin{array}{l}\text { Frecuencia de } \\
\text { la tasa de tuber- } \\
\text { culosis entre los } \\
\text { trabajadores del } \\
\text { Hospital Antonio } \\
\text { Pedro. }\end{array}$ & $\begin{array}{l}\text { Describir la tasa } \\
\text { de frecuencia de } \\
\text { positividad de } \\
\text { tuberculosis entre } \\
\text { los trabajadores } \\
\text { de un hospital } \\
\text { universitario de la } \\
\text { ciudad de Niterói, } \\
\text { Río de Janeiro, en } \\
\text { el período enero } \\
2008 \text { y marzo de } \\
2011 .\end{array}$ & $\begin{array}{l}\text { Estudio observacional, } \\
\text { transversal analítico. A } \\
\text { partir de una base de } \\
\text { datos proporcionada } \\
\text { por el programa de } \\
\text { control de tuberculosis } \\
\text { del hospital se indaga- } \\
\text { ron a los trabajadores } \\
\text { de salud que se realiza- } \\
\text { ron la PPD entre enero } \\
2008 \text { a marzo } 2011 .\end{array}$ & $\begin{array}{l}\text { Del total de } 663 \text { empleados, } 261 \text { (39.0\%) mostra- } \\
\text { ron positividad en la prueba tuberculina, siendo } \\
45.0 \% \text { hombres. Los sectores con las mayores tasas } \\
\text { de positividad tuberculina fueron los servicios de } \\
\text { nutrición (12.0\%) y de emergencia ( } 13.0 \%) \text {, donde } \\
\text { la tasa de conversión entre los profesionales fue de } \\
10.0 \% \text { en } 3 \text { años. }\end{array}$ \\
\hline 7 & Hernández et al. & 2014 & $\begin{array}{l}\text { Pesquisa de } \\
\text { infección tuber- } \\
\text { culosa latente en } \\
\text { personal de la } \\
\text { salud en cuatro } \\
\text { instituciones de } \\
\text { salud en Santia- } \\
\text { go de Chile. }\end{array}$ & $\begin{array}{l}\text { Determinar la } \\
\text { prevalencia de } \\
\text { infección tuber- } \\
\text { culosa latente en } \\
\text { funcionarios de la } \\
\text { salud en diferentes } \\
\text { áreas laborales de } \\
\text { riesgo. }\end{array}$ & $\begin{array}{l}\text { Estudio observacio- } \\
\text { nal, descriptivo en } 76 \\
\text { profesionales de salud } \\
\text { de laboratorio clínico } \\
\text { del área de atención } \\
\text { respiratoria de Cuatro } \\
\text { hospitales de Santiago. } \\
\text { Se realizó la prueba } \\
\text { IGRA. }\end{array}$ & $\begin{array}{l}\text { Se evidenció infección tuberculosa latente en } 20 \\
\text { de las } 76 \text { ( } 26.3 \%) \text { personas estudiadas. En aquellos } \\
\text { con antecedente de contacto en el pasado con en- } \\
\text { fermos de tuberculosis, la positividad del test llegó } \\
\text { a } 62.5 \% \text {; en aquellos que pertenecían al Programa } \\
\text { Nacional de Control de la Tuberculosis, a } 50 \% \text { y } \\
\text { en los que realizaban toma de esputo inducido, } \\
\text { baciloscopías o cultivo de micobacterias, a 38.0\%. } \\
\text { La proporción de individuos con QFT positivo fue } \\
\text { significativamente menor en aquellos funcionarios } \\
\text { que no tenían estos antecedentes ( } 15.7 \% \text {, p = 0.03). }\end{array}$ \\
\hline
\end{tabular}


Tabla 2. Continuación

\begin{tabular}{|c|c|c|c|c|c|c|}
\hline 8 & Borges et al. & 2011 & $\begin{array}{l}\text { Prevalencia } \\
\text { de infección } \\
\text { latente por } \\
\text { Mycobacterium } \\
\text { tuberculosis en } \\
\text { profesionales de } \\
\text { la red básica de } \\
\text { salud. }\end{array}$ & $\begin{array}{l}\text { Estimar la preva- } \\
\text { lencia de infección } \\
\text { latente por } M \text {. } \\
\text { tuberculosis e iden- } \\
\text { tificar las caracterís- } \\
\text { ticas relacionadas a } \\
\text { la infección latente } \\
\text { de los trabajadores } \\
\text { de la red básica de } \\
\text { salud. }\end{array}$ & $\begin{array}{l}\text { Estudio observacional, } \\
\text { descriptivo analítico en } \\
137 \text { trabajadores de la } \\
\text { red básica de } \\
\text { salud que desarrollaban } \\
\text { sus actividades en un } \\
\text { municipio de Brasil. } \\
\text { Se realizó la PPD y } \\
\text { cuestionario. }\end{array}$ & $\begin{array}{l}\text { La prevalencia estimada de infección latente de M. } \\
\text { tuberculosis de los trabajadores sanitarios evalua- } \\
\text { dos fue del 32.8\%. No ha sido posible relacionar las } \\
\text { características estudiadas con la infección latente } \\
\text { entre los trabajadores de la red básica de salud, a } \\
\text { excepción del tabaquismo con un OR: 3,03; IC } 95 \% \\
\text { 1,05-8,77). }\end{array}$ \\
\hline 9 & Soto et al. & 2017 & $\begin{array}{l}\text { Prevalencia de } \\
\text { infección tuber- } \\
\text { culosa latente } \\
\text { en trabajadores } \\
\text { de salud de } \\
\text { establecimientos } \\
\text { del primer nivel } \\
\text { de atención. } \\
\text { Lima, Perú. }\end{array}$ & $\begin{array}{l}\text { Estimar la prevalen- } \\
\text { cia de ITL en TS de } \\
\text { establecimientos } \\
\text { del primer nivel de } \\
\text { atención, utilizando } \\
\text { los datos de la } \\
\text { vigilancia centinela } \\
\text { realizada en los ES } \\
\text { del primer nivel } \\
\text { de atención de la } \\
\text { RED Bonilla de la } \\
\text { Dirección Regional } \\
\text { de Salud (DIRESA) } \\
\text { del Callao, así como } \\
\text { describir los factores } \\
\text { de riesgo que mejor } \\
\text { predicen la ITL en } \\
\text { esta población. }\end{array}$ & $\begin{array}{l}\text { Estudio observacional, } \\
\text { transversal analítico en } \\
150 \text { trabajadores de } \\
\text { salud. } \\
\text { La prevalencia de ITL } \\
\text { se determinó de acuer- } \\
\text { do con el resultado } \\
\text { positivo obtenido con } \\
\text { IGRA. }\end{array}$ & $\begin{array}{l}\text { La prevalencia de ITL en trabajadores de salud } \\
\text { fue } 56.0 \% \text {. En trabajadores con más de } 10 \text { años } \\
\text { de servicio la prevalencia se incrementó a } 63.0 \% \\
\text { y en trabajadores con más de } 35 \text { años de servicio } \\
\text { se encontraron prevalencias entre } 58.0 \text { y } 60.0 \% \text {. El } \\
\text { mayor tiempo de servicio fue uno de los principales } \\
\text { factores de riesgo. }\end{array}$ \\
\hline 10 & Bavaresco et al. & 2017 & $\begin{array}{l}\text { Tuberculosis } \\
\text { latente entre } \\
\text { profesionales } \\
\text { de un hospital } \\
\text { oncológico de } \\
\text { referencia. Latent } \\
\text { tuberculosis } \\
\text { among profes- } \\
\text { sionals from a } \\
\text { referral hospital } \\
\text { in oncology }\end{array}$ & $\begin{array}{l}\text { El objetivo principal } \\
\text { fue estimar la } \\
\text { prevalencia de TBL } \\
\text { entre el personal } \\
\text { clínico y adminis- } \\
\text { trativo de un hos- } \\
\text { pital oncológico de } \\
\text { referencia en Río } \\
\text { Grande. El objetivo } \\
\text { secundario fue } \\
\text { evaluar la tasa de } \\
\text { conversión del test } \\
\text { de tuberculina y los } \\
\text { factores de riesgo } \\
\text { en esta población } \\
\text { con PPD positiva. }\end{array}$ & $\begin{array}{l}\text { Estudio observacional, } \\
\text { transversal analítico en } \\
225 \text { profesionales en } \\
2013 \text { a quienes se les } \\
\text { aplicó PPD y medición } \\
\text { de factores de riesgo. A } \\
\text { los que resultaron nega- } \\
\text { tiva se les volvió aplicar } \\
\text { PPD en } 2014 \text { para medir } \\
\text { la tasa de conversión en } \\
1 \text { año. Se tomó como } \\
\text { positivo con tamaño } \\
\text { igual o mayor a } 10 \text { mm } \\
\text { de induración. }\end{array}$ & $\begin{array}{l}\text { El } 60.0 \% \text { tuvo PPD positiva; el 78.2\% fueron mujeres } \\
\text { y la mayoría de los positivos habían trabajado en } \\
\text { el hospital } 4 \text { años o menos. A los que tuvieron PPD } \\
\text { negativa en } 2013 \text { se les realizó otra vez en 2014, } \\
\text { obteniendo una tasa de conversión del } 9.37 \% \text {. No } \\
\text { hubo diferencia significativa de la prevalencia de } \\
\text { TBL entre la categorías de profesionales, y no se } \\
\text { encontró asociación de los factores de riesgo a ITBL. }\end{array}$ \\
\hline 11 & Nasreen et al. & 2016 & $\begin{array}{l}\text { Prevalencia de } \\
\text { TBL en trabaja- } \\
\text { dores de salud } \\
\text { de países con } \\
\text { elevada carga de } \\
\text { TB: Una revisión } \\
\text { sistemática y } \\
\text { metaanálisis. }\end{array}$ & $\begin{array}{l}\text { Estimar la preva- } \\
\text { lencia de TBL entre } \\
\text { trabajadores de } \\
\text { salud de países } \\
\text { con elevada carga } \\
\text { de TB. }\end{array}$ & $\begin{array}{l}\text { Metaanálisis, revisando } \\
\text { estudios de TBL en } \\
\text { trabajadores de salud } \\
\text { de países con alta carga } \\
\text { de TB } \\
\text { Se revisaron bases: ME- } \\
\text { DLINE (Ovid), EMBASE } \\
\text { (Ovid), CINAHL (Ovid) } \\
\text { and ISI Web of Science } \\
\text { (Thompson-Reuters) } \\
\text { y literatura gris fue } \\
\text { utilizada en lenguaje } \\
\text { inglés. Se utilizaron los } \\
\text { términos MeSH: "LTBI"y } \\
\text { "health care providers". }\end{array}$ & $\begin{array}{l}\text { La prevalencia agrupada de TBL fue de } 47.0 \% \\
\text { (05\% IC } 34 \% \text {-60\%). El país con menor prevalencia } \\
\text { agrupada fue Brasil, con un 37.0\% y Sudáfrica tuvo } \\
\text { la más alta con un 64.0\%. La prevalencia de TBL en } \\
\text { estudiantes de medicina y enfermería fue del } 26.0 \% \\
\text { (95\% IC 6\%-46\%, I2=99.3\%). La prevalencia de TBL } \\
\text { entre todas las categorías de trabajadores de salud } \\
\text { fue de } 57.0 \% \text { (95\% Cl } 44 \% \text { a } 70 \% \text {, I2 = 99.1\%). La } \\
\text { incidencia se midió solo en } 4 \text { países. Se encontró } \\
\text { una incidencia acumulada del 2.8\% entre estudian- } \\
\text { tes de medicina de Brasil; y en Sudáfrica se reportó } \\
\text { una incidencia acumulada de todos los tipos de } \\
\text { trabajadores de salud del } 38.0 \% \text {. }\end{array}$ \\
\hline
\end{tabular}


Tabla 2. Continuación

Tabla 2. Continuación.

2012

Infección

tuberculosa en

trabajadores de

salud de aten-

ción primaria.

13

González et al. $\quad 2010$

Tuberculosis en trabajadores de salud.
Estimar la prevalen- Se realizó un estudio

cia y determinar el descriptivo transverriesgo de factores sal. Se administró un asociados con TBL, cuestionario para en trabajadores de recolección de datos salud de atención primaria en la ciudad de Vitoria Brasil.

Evaluar la problemática de TBL en personal de salud a través de una experiencia de prevención.

sociodemográficos, reacción de $10 \mathrm{~mm}$ a 2 UT PPD en $\geq$. Se de prevalencia de la características clínicas

y conocimientos sobre

TB; se aplicó PPD, la cual fue leída a las 72 horas posterior a la aplicación midiendo la induración.

\section{La infección de TB se} definió mediante una realizaron dos estudios infección tuberculosa: uno en 1998 y otro en 2008.

\begin{tabular}{ll}
\hline $14 \quad$ Argüello et al. $2017 \quad$ Manejo de la \\
& infección tuber- \\
& culosa latente en \\
& trabajadores de \\
& la salud: 10 años \\
& de experiencia \\
& en un solo \\
& centro.
\end{tabular}
sión retrospectiva de los registros de los servicios de salud y bienestar del empleado de todos los trabajadores de la salud diagnosticados con

LTBI. A los profesionales
Se llevó a cabo una revide salud diagnosticados con LTBI se les ofreció isoniazida (INH) de 9 meses, rifampicina de 4 meses (RIF), rifapentina/ isoniazida (RPT/INH) semanal durante 12 semanas o ningún tratamiento. La aceptación, las tasas de finalización y los efectos secundarios se informaron para cada régimen.

\begin{tabular}{lll}
\hline $2016 \quad$ Ochoa et al. & Prevalencia & Prevalencia de \\
& de infección & infección tuber- \\
& tuberculosa en & culosa latente en \\
& trabajadores de & trabajadores de \\
& la salud de la red & la salud (PS) de la \\
& de hospitales & red de hospitales \\
& públicos en Me- & públicos en Me- \\
& dellín, Colombia: & dellín, Colombia, \\
& un enfoque & 2013-2015. \\
& bayesiano. & \\
&
\end{tabular}
de prevalencia de la infección de tuberculosis latente (LTBI) mediante la prueba cutánea de tuberculina (TST) y la prueba de cuantificación (QFT) en 1218 trabajadores de la salud en Medellín, Colombia, entre 2013 a 2015.

Se realizó una encuesta

El 39.4\% de los participantes presentó PPD mayor o igual a $10 \mathrm{~mm}$. Solo en las variables de ser fumador y PPD previa positiva se encontraron una asociación estadísticamente significativa con TBL.

La prevalencia en 1998 fue de $31.7 \%$ y en 2008 de $35.0 \%$. A pesar de las limitaciones en el recurso humano para realizar estudios de incidencia de TB, la protección personalizada, la educación en TB y la quimioprofilaxis podrían reducir los riesgos de TB en TS.

Entre 2005 y 2014, 363 de 927 (39.0\%) trabajadores sanitarios con diagnóstico de LTBI aceptaron el tratamiento. De 363, 202 eligieron INH, 106 RIF y 55 RPT / INH. Las tasas de finalización para cada régimen fueron $58.0 \%, 80.0 \%$ y $87.0 \%$, respectivamente.

\begin{abstract}
Se construyó un modelo de clases latentes utilizando un enfoque bayesiano con antecedentes informativos sobre la sensibilidad y la especificidad de la TST. La proporción de resultados concordantes (TST+, QFT+) fue del $41.0 \%$ y los resultados discordantes contribuyeron con el 27.0\%. La estimación marginal de la prevalencia $\mathrm{P}(\mathrm{LTB}+$ ) fue de $62.1 \%$ [intervalo creíble (Crl) del $95 \%$ 53.0-68.2]. La probabilidad de LTBI+ de obtener resultados positivos en ambas pruebas fue del 99.6\% (Crl del $95 \%$ 98.1-99.9). La sensibilidad fue de 88.5 para la TST y 74.3 para la QFT, y la especificidad fue de 87.8 para la TST y 97.6 para la QFT. Se encontró una alta prevalencia de LTBI en los PCS con exposición acumulada en el tiempo en los hospitales que carecen de planes de control.
\end{abstract}


No obstante, los estudios de esta revisión no son concluyentes al respecto y no se puede olvidar que la infección depende de varios factores, siendo el estado inmunitario el principal ${ }^{10}$.

Es necesario realizar estudio de mayor profundidad en este tema, ya que el riesgo que supone trabajar de cerca de población enferma de tuberculosis ya sea en contacto directo con los pacientes enfermos, a través del estudio de muestras o en ambientes laborales inadecuados, ha llevado a realzar el tema de la ITBL al grado de ser incluida en el listado internacional de enfermedades ocupacionales.

En El Salvador, existe limitada información acerca de la ITBL en trabajadores en salud. No se encontraron publicaciones indexadas al respecto. Aun así, entre la información disponible se encuentra una presentación realizada en el XI Congreso Nacional de Tuberculosis en El Salvador22; se notificaron casos de TB en auxiliares de servicios, enfermeras y médicos. Además, en una tesis de grado se reporta que la prevalencia de TB en el personal de salud de la región metropolitana es de 2 a 5 veces mayor que en la población general23.

\section{Conclusión}

La prevalencia de tuberculosis sigue siendo mayor en los trabajadores de salud que en la población general. Esto afecta mayoritariamente a las mujeres entre los 35 y 43 años que han laborado por más de 10 años en centros penitenciarios o centros de atención primaria.

\section{Referencias Bibliográficas}

1. Organisation Mondiale de la Santé. Global tuberculosis report 2018. 2018.

2. OPS/OMS. Unidos para poner fin a la tuberculosis [Internet]. Pan American Health Organization / World Health Organization. 2016 [Citado el 15 de enero de 2020]. Disponible en: https://bit.ly/2O3WZ1w

3. Dye C, Scheele S, Dolin P, Pathania V, Raviglione MC. WHO Global Surveillance and Monitoring Project. Global Burden of Tuberculosis: Estimated Incidence, Prevalence, and Mortality by Country. JAMA. 1999; 282(7):677. DOI: https://doi. org/10.1001/jama.282.7.677

4. OPS/OMS. Tuberculosis - Datos Generales. [Internet]. Pan American Health Organization / World Health Organization. 2016 [Citado el 17 de agosto de 2019]. Disponible en: https://bit.ly/3aRFBae
5. International Labour Office. List of occupational diseases: identification and recognition of occupational diseases: criteria for incorporating diseases in the ILO list of occupational diseases. Rev. 2010. Geneva: International Labour Office; 2010. 72 p.

6. CDC. Factores de riesgo de la tuberculosis. Datos básicos sobre la tuberculosis. [Internet]. Center for Disease Control and Prevention 2018. [Citado el 15 de enero de 2020]. Disponible en: https://bit.ly/2t3hQuF

7. Ministerio de Salud de El Salvador. Lineamientos técnicos para la prevención y control de la Tuberculosis. 2015. 110 p.

8. Sabri A, Quistrebert J, Naji Amrani H, Abid A, Zegmout A, Abderrhamani Ghorfi l, et al. Prevalence and risk factors for latent tuberculosis infection among healthcare workers in Morocco. PLOS ONE. 2019;14(8). DOI: https://doi.org/10.1371/journal. pone.0221081

9. Baussano I, Nunn P, Williams B, Pivetta E, Bugiani M, Scano F. Tuberculosis among Health Care Workers. Emerg Infect Dis. 2011; 17(3):488-94. DOI: https://doi.org/10.3201/ eid1703.100947

10. OMS. Directrices sobre la atención de la infección tuberculosa latente. [Internet]. Organización Mundial de la Salud. [Citado el 12 de noviembre de 2019]. Disponible en: https://bit.ly/2GuCBCs

11. Prado TN, Riley LW, Sanchez M, Fregona G, Nóbrega RLP, Possuelo LG, et al. Prevalence and risk factors for latent tuberculosis infection among primary health care workers in Brazil. Cad Saúde Pública. 2017; 33:e00154916. DOI: https://doi. org/10.1590/0102-311×00154916

12. Soto Cabezas MG, Munayco Escate CV, Chávez Herrera J, López Romero SL, Moore D. Prevalencia de infección tuberculosa latente en trabajadores de salud de establecimientos del primer nivel de atención. Lima, Perú. Rev Peru Med Exp Salud Pública. 2017; 34(4):649. DOI: https:// doi.org/10.17843/rpmesp.2017.344.3337

13. Ochoa J, León AL, Ramírez IC, Lopera CM, Bernal E, Arbeláez MP. Prevalence of tuberculosis infection in healthcare workers of the public hospital network in Medellín, Colombia: a Bayesian approach. Epidemiol Infect. 2017; 145(6):1095-106. DOI: https:// doi.org/10.1017/S0950268816003150

14. Busatto $C$, Nunes $L$ de $S$, Valim AR de $M$, Valença MS, Krug SF, Becker D, et al. Tuberculosis among prison staff in Rio Grande do Sul. Rev Bras Enferm. 2017; 70(2):370-5. DOl: https://doi. org/10.1590/0034-7167-2016-0012

15. Nasreen S, Shokoohi M, Malvankar-Mehta MS. Prevalence of Latent Tuberculosis among Health Care Workers in High Burden Countries: A Systematic Review and Meta- 
Analysis. Wilkinson KA, editor. PLoS ONE. 2016; 11(10):e0164034. DOI: https://doi. org/10.1371/journal.pone.0164034

16. Gutiérrez DSB, Molina DDM. Riesgo de ocurrencia de la tuberculosis en los trabajadores del Hospital Universitario Neumológico Benéfico Jurídico de La Habana. Rev Cubana Med Trop. 2012; 64(1): 55-60.

17. Silvana Borges T, Chaida Sonda E, Daronco A, Battisti F, Moura Baptista dos Santos M, Moura Valim AR, et al. Prevalência de infecção latente por mycobacterium tuberculosis em profissionais da rede básica de saúde. Rev Bras Em Promoção Saúde. 2014; 27(2):269-75. DOI: https://doi. org/10.5020/18061230.2014.p269

18. Bavaresco ACW, Busatto C, Reis AJ, Krug SF, Segatto N, Valim AR de M, et al. Latent tuberculosis among professionals from a referral hospital in oncology. Clin Biomed Res. 2017; 37(4):281-7. DOl: https://doi. org/10.4322/2357-9730.75447

19. Lacerda TC, Souza FM, Prado TN, Locatelli RL, Fregona G, Lima R de CD, et al. Tuberculosis infection among primary health care workers. J Bras Pneumol. 2017; 43(6):41623. DOI: https://doi.org/10.1590/s1806$\underline{37562016000000211}$
20. Rogerio WP, Prado TN, Souza FM, Pinheiro J dos S, Rodrigues PM, Sant'anna AP do $\mathrm{N}$, et al. Prevalência e fatores associados à infecção pelo Mycobacterium tuberculosis entre agentes comunitários de saúde no Brasil, usando-se a prova tuberculínica. Cad Saúde Pública. 2015; 31 (10):2199210. DOI: https://doi.org/10.1590/0102311X00152414

21. Cohen A, Mathiasen VD, Schön T, Wejse C. The global prevalence of latent tuberculosis: a systematic review and meta-analysis. Eur Respir J. 2019; 54(3):1900655. DOI: https:// doi.org/10.1183/13993003.00655-2019

22. Andreu $\mathrm{H}$. Enfoque de la TB y su abordaje desde el ámbito laboral en El Salvador. [Internet]. 2016. [Citado el 16 de noviembre de 2019]. Disponible en: https://bit. ly/30YX77L

23. Figueroa Cañas R. Prevalencia, caracterización clínica y epidemiológica de la tuberculosis en personal de salud en El Salvador. [Internet]. 2019. [Citado el 16 de noviembre de 2019]. Disponible en: https:// bit.ly/2tK1s2B 